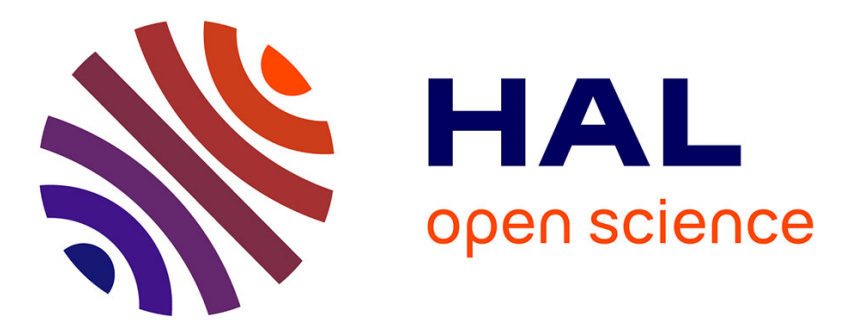

\title{
First principles investigation of the crystal and electronic structures of $\mathrm{CeNCl}$ and "CeNF"
}

Samir F. Matar

\section{To cite this version:}

Samir F. Matar. First principles investigation of the crystal and electronic structures of $\mathrm{CeNCl}$ and "CeNF". Solid State Sciences, 2015, 48, pp.1-6. 10.1016/j.solidstatesciences.2015.06.009 . hal01172410

\author{
HAL Id: hal-01172410 \\ https://hal.science/hal-01172410
}

Submitted on 7 Jul 2015

HAL is a multi-disciplinary open access archive for the deposit and dissemination of scientific research documents, whether they are published or not. The documents may come from teaching and research institutions in France or abroad, or from public or private research centers.
L'archive ouverte pluridisciplinaire HAL, est destinée au dépôt et à la diffusion de documents scientifiques de niveau recherche, publiés ou non, émanant des établissements d'enseignement et de recherche français ou étrangers, des laboratoires publics ou privés. 


\title{
First principles investigation of the crystal and electronic structures of $\mathrm{CeNCl}$ and "CeNF"
}

\author{
Samir F. Matara,b,* \\ a CNRS, ICMCB, UPR 9048, F-33600 Pessac, France. \\ b Univ. Bordeaux, ICMCB, UPR 9048, F-33600 Pessac, France. \\ E_mail: samir.matar@icmcb.cnrs.fr \& abouliess@gmail.com
}

Abstract:

Based on DFT energy discrimination and detailed electronic and bonding properties, it is shown that the hitherto unexplored cerium nitride fluoride can be obtained in rhombohedral ThNF structure whereas actual CeNCl is confirmed in the early determination PbFCl-like structure. The respectively adopted $3 \mathrm{D}$ and $2 \mathrm{D}$ like structures are assigned to the major role played by the F polarizability, four times smaller than $\mathrm{Cl}$. In their ground state both compound are found small gap insulators with $1.9 \mathrm{eV}(\mathrm{CeNCl}), 1.8 \mathrm{eV}$ (CeNF) compared to insulating isoelectronic cerine $\mathrm{CeO}_{2}$ with $2.2 \mathrm{eV}$ band gap. The bonding shows balance between covalence brought by $\mathrm{Ce}-\mathrm{N}$ bond versus ionic $\mathrm{Ce}-\mathrm{F}(\mathrm{Cl})$ bonding and $d(\mathrm{Ce}-\mathrm{N})<d(\mathrm{Ce}-\mathrm{F}(\mathrm{Cl}))$ distances. Synthesis routes for CeNF are proposed.

Keywords: Mixed-anions, crystal structure, iono-covalent, DFT, COOP. 


\section{Introduction}

The search of mixed anions compounds is an active field of research in so far that they offer original structural, physical and chemical properties related with the ionocovalent bonds. Such compositions refer on one hand to single anions mixtures as in layered $\mathrm{PbFCl}$-type structure of $\mathrm{BiOF}$, known for its use in ionic conductor solid electrolytes [1] as well as in more complex recently identified $\mathrm{Ba}_{18} \mathrm{~F}_{18} \mathrm{In}_{8} \mathrm{~S}_{21}$ and $\mathrm{Ba}_{9} \mathrm{~F}_{10} \mathrm{In}_{4} \mathrm{~S}_{10}$ low dimensional sulfide-fluorides [2] and on the other hand to poly-anionic constituents as phosphate anion associated to molybdate and tungstate anions in $\mathrm{Rb}_{2} \mathrm{Bi}\left(\mathrm{PO}_{4}\right)\left(\mathrm{MO}_{4}\right), \mathrm{M}=\mathrm{Mo}, \mathrm{W}$ [3]. In this context nitride-halides of formulation $A^{\mathrm{IV}} \mathrm{N} X(X$ : halogen) can be considered as pseudo-oxides being isoelectronic with $A^{\mathrm{IV}} \mathrm{O}_{2}$. $A^{\mathrm{IV}}$ stands for a tetravalent element such as a transition metal in Ti ${ }^{\mathrm{IV} N F}$ [4] and $\mathrm{Zr}^{\mathrm{IV}} \mathrm{NF}$ [5]. Most of nitride-halides are single member, mainly with $X=\mathrm{F}$. However in the case of $\mathrm{Th}^{\mathrm{IV}}$ the whole ThN $X$ series exists, i.e. for $X=\mathrm{F}, \mathrm{Cl}, \mathrm{Br}$, I [6]. Interestingly, ThNF is rhombohedral while the other ThNX are tetragonal with a PbFCl-like structure. This structure is also adopted by $\mathrm{CeNCl}$ which is the only rare earth nitride halide [7]. Recent review on layered nitride halides $\mathrm{MN} X(\mathrm{M}=\mathrm{IV} B$ transition element and $X=\mathrm{Cl}, \mathrm{Br}, \mathrm{I})$ with orthorhombic FeOCl -type structure point out to superconducting properties evidenced upon partial intercalation with alkali elements [8].

Electronic structure, phase stability from energy criteria and chemical bonding properties are nowadays accessible with quantum theoretical density functional theory DFT $[9,10]$ based methods. In recent years such methodology was used to investigate the physical properties including magnetic ones of nitride fluorides [11-14]. For instance CrNF [14] was found to mimic the well known rare room temperature ferromagnetic oxide: $\mathrm{CrO}_{2}$ used in high density magnetic recording, with major difference of magnetic response to pressure characterizing $\mathrm{CrNF}$ as a soft ferromagnet versus hard magnetic $\mathrm{CrO}_{2}$. Herein we extend the investigation to the study of $\mathrm{CeNCl}$ and propose the hitherto unidentified CeNF. Particularly the structure of $\mathrm{CeNCl}$ is revisited by complementing the partial early determination [7] with calculated positional parameters of $\mathrm{Ce}$ and $\mathrm{Cl}$ and providing a description of its electronic structure and bonding properties. Further, based on ThNF former works, a proposition of a ground state structure for CeNF is given from energy quantities determined for two model structures: rhombohedral versus tetragonal. 
For these objectives we used two computational methods within DFT: The VASP code with PAW (projected augmented plane wave) method [15,16] allows geometry optimization, ground state energies and analyses of the charge density. Then the full analysis of the properties of the electronic structure and chemical bonding properties based on the overlap populations (COOP criterion) was done using full potential scalarrelativistic augmented spherical wave (ASW) method [17]. The PBE generalized gradient approximation (GGA) functional [18], accounting for the effects of exchange and correlations within DFT, was considered in all calculations. In spite of the localized $f$ character of cerium, the calculations did not require using GGA+U schemes for better results. For details on the methods the reader is referred to the theoretical development in a review [19].

\section{Crystal chemistry and discussion.}

Like ThN $X(X=\mathrm{Cl}, \mathrm{Br}, \mathrm{I})$, CeNCl crystallizes in tetragonal PbFCl-type structure with P4/nmm space group. The structure sketched at Fig. 1a is characterized by layer like $[\mathrm{CeN}]_{\mathrm{n}}$ with nitrogen atoms forming a square lattice Short Ce-N bonds, likely to exhibit covalent character, are opposed to longer $\mathrm{Ce}-\mathrm{Cl}$ bonds. $[\mathrm{CeN}]_{\mathrm{n}}$ sheets are separated by puckered chlorine double layers and $\mathrm{Ce}$ is found in a monocapped square planar antiprism $\left\{\mathrm{N}_{4} \mathrm{Cl}_{5}\right\}$ formed by four equatorial $\mathrm{Cl}\left(\mathrm{Cl}_{\text {eq. }}\right)$ and one apical $\mathrm{Cl}\left(\mathrm{Cl}_{\text {ap. }}\right)$.

For $X=\mathrm{F}$ characterizing $\mathrm{Th}^{\mathrm{IV}} \mathrm{NF}$, the structure is rhombohedral with $R \overline{3} m$ space group. Oppositely to $\mathrm{PbFCl}-$ type, it has a rather three dimensional structure whose projection in hexagonal symmetry ( 6 atoms of each species, i.e. 3 times more than in rhombohedral symmetry) reported for Ce case under focus here, is shown in Fig. 1b. The rare earth is surrounded by $4 \mathrm{~N}$ and $4 \mathrm{~F}$ (see the bonds around Ce designated by ' 1 ') forming a distorted cube due to longer Ce-F versus shorter Ce-N (cf. Table 2).

The difference between the two structures shows that dimensionality is a key parameter driven by the nature of the halide: Chlorine is a big anion and subsequently largely polarizable $\left(\alpha=2.18 \AA^{3}\right)$ [20]. It tends to reduce its negative charge by polarizing (distorting its electron cloud) towards the cation (Ce), whence the layer-like conformation as in $\mathrm{PbFCl}$ type. On the contrary this cannot be obtained with smaller size fluorine which is much less polarizable $\left(\alpha=0.557 \AA^{3}\right)$ [20] and the resulting structure 
cannot have a 2D character. This is quantitatively shown with the discussion of the electronic structures.

\section{Geometry optimization, energy and electron localization: Results and} discussions.

The experimental structure determination of $\mathrm{CeNCl}$ provides only $a$ and $c$ tetragonal parameters (Table 1). As shown in Fig. $1 \mathrm{a}, \mathrm{N}$ is at the origin whereas Ce and $\mathrm{Cl}$ are at $(2 c)$ particular positions $(0,1 / 2, z)$ for which we chose as starting trial $z$ coordinates those of ThNCl, i.e. 0.635 and 0.165 for $\mathrm{Cl}$ and Ce respectively [13]. The resulting parameters and volume after full geometry optimization are in fair agreement with experiment with nevertheless a trend of a $2 \%$ smaller volume for CeNCl. $z(\mathrm{Ce})$ and $z(\mathrm{Cl})$ are also within range of starting values of $\mathrm{ThNCl}$. The volume of CeNF is found smaller due to the size difference between $\mathrm{Cl}$ and $\mathrm{F}$ (1.0 versus $0.7 \AA$ ). However a more striking feature is the large diminution of the $\mathrm{c} / \mathrm{a}$ ratio which decreases from $1.73 \mathrm{in} \mathrm{CeNCl}$ to $1.55 \mathrm{in} \mathrm{CeNF}$; the consequence is a small difference $d(C e-F)-d(C e-N)=0.13 \AA$, versus $d(C e-C l)-d(C e-N)=$ $0.88 \AA$. Such a result is not due to the radii of atomic $\mathrm{N}$ and $\mathrm{F}$ which are close in magnitudes (resp. 0.75 and $0.72 \AA$ ), but mainly arises from the reduced c/a. This is likely related with the probable instability of CeNF in such layer like structure as shown in next paragraph from energy trends. The total energies per 2 formula units (FU) are provided at the last line of Table 1; they are used in next paragraph for a confrontation with the rhombohedral structure energy results.

Although CeNCl is tetragonal as described above, subsequent calculations on both CeNCl and CeNF in the rhombohedral ThNF structure were carried out in order to energy discriminate the trial structures. Table 2 shows the results considering the crystal data of rhombohedral ThNF as starting input [13]. For the nitride chloride, a largely smaller volume is calculated with respect to its experimental one whereas a similar volume magnitude is obtained for CeNF. The interatomic distances are consequently smaller but the difference $d(\mathrm{Ce}-\mathrm{Cl})-\mathrm{d}(\mathrm{Ce}-\mathrm{N})$ is now $0.63 \AA$, versus $0.88 \AA$ in the tetragonal structure. The trend $d(C e-F)-d(C e-N)=0.24 \AA$ is larger than in the tetragonal structure.

The last line gives the total energies for the two compounds. A comparison with the energies in Table 1 shows the following trend: 


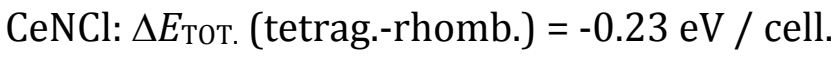

CeNF: $\Delta E_{\text {TOT. }}$ (tetrag.-rhomb.) $=+0.65 \mathrm{eV} /$ cell.

$\mathrm{CeNCl}$ is stable in its experimentally evidenced ground state tetragonal structure. This validates the calculations in reproducing the experimental structure. More interesting is the large stabilization of CeNF in the rhombohedral structure versus the tetragonal one.

Lastly we have carried complementary calculations testing other structural configurations:

- Calculation of $\mathrm{CeNCl}$ in the orthorhombic FeOCl-type adopted by layered nitride halides $\mathrm{MN} X(\mathrm{M}=\mathrm{IV} B$ transition element and $X=\mathrm{Cl}, \mathrm{Br}, \mathrm{I})$ as discussed in ref. [8]. The energy of the fully geometry optimized structure is then $-41.935 \mathrm{eV}$, i.e. $1.41 \mathrm{eV}$ higher than the ground state structure. Then among the nitride halides tetragonal CeNCl presents unique structural features.

- In view of the three dimensional like rhombohedral CeNF structure and the closer magnitude of $d(\mathrm{Ce}-\mathrm{F}) / \mathrm{d}(\mathrm{Ce}-\mathrm{N})$ with respect of the tetragonal setting, an anionic disorder was considered by redistributing the anions over the four available sites (cf. Table 2). The result is a large energy destabilization by $\sim 1.8 \mathrm{eV} /$ cell versus the anionically ordered structure.

The results can be assessed further by analyzing the charge density issued from the self consistent calculations using the AIM (atoms in molecules theory) approach [21] developed by Bader whereby the molecules are partitioned into atoms as based on the electronic charge density whereby it is assumed that in chemical systems, charge density reaches a minimum between atoms. The Bader analysis does not constitute a tool for evaluating absolute ionizations but it is useful when trends between similar chemical compounds are discussed. The results of computed charges $Q$ are such that they lead to neutrality:

rh. CeNF: $Q(\mathrm{Ce})=+2.15 ; Q(\mathrm{~N})=-1.37 ; Q(\mathrm{~F})=-0.78 . \Delta Q= \pm 2.15$

tet. CeNF: $Q(\mathrm{Ce})=+2.09 ; Q(\mathrm{~N})=-1.28 ; Q(\mathrm{~F})=-0.81 . \Delta Q= \pm 2.09$ 
and

rh. CeNCl: $\quad \mathrm{Q}(\mathrm{Ce})=+1.96 ; \mathrm{Q}(\mathrm{N})=-1.32 ; \mathrm{Q}(\mathrm{Cl})=-0.64 . \quad \Delta \mathrm{Q}= \pm 1.96$

tet. CeNCl: $\quad \mathrm{Q}(\mathrm{Ce})=+2.00 ; \mathrm{Q}(\mathrm{N})=-1.29 ; \mathrm{Q}(\mathrm{Cl})=-0.71 . \quad \Delta \mathrm{Q}= \pm 2.00$

While charge flows from Ce towards $\mathrm{N}$ and $X$ in both compounds and structures, the amount of charge transfer does not lead to the formal $\mathrm{Ce}^{+4} \mathrm{~N}^{-3} X^{-1}$. Such a fully ionic character cannot be obtained in the solid state but the respective charges are proportional to the ionic magnitudes, with larger charge on $\mathrm{N}$ than $X$ and an overall more ionic CeNF than $\mathrm{CeNCl}$ as expected from their respective electronegativities: $\mathrm{F}(\chi=$ 3.98) and $\chi(\mathrm{Cl})=3.16$. The rhombohedral structure of CeNF allows for larger charge transfer from Ce to $\mathrm{N}$ and $\mathrm{F}$ versus the tetragonal one. The opposite trend is observed in $\mathrm{CeNCl}$ in its ground state tetragonal structure. These trends are similar to ThN $X$ cases [13] though with larger charge transfer magnitudes due to difference in electronegativities: $\chi(\mathrm{Th})=1.3$ while $\chi(\mathrm{Ce})=1.12$. Then besides the energy based criteria above, the rhombohedral structure is preferred for CeNF because it allows larger charge transfer on $\mathrm{N}$ ensuring for the strong Ce- $\mathrm{N}$ bonding while keeping a constant negative charge on $\mathrm{F}$ close to full anionic.

The charge transfers in isoelectronic $\mathrm{CeO}_{2}$ are calculated for the sake of comparison with the two ternary compounds:

Fluorite $\mathrm{CeO}_{2}: \mathrm{Q}(\mathrm{Ce})=+2.24 ; \mathrm{Q}(0)=-1.12$.

With $\Delta \mathrm{Q}= \pm 2.24$, the oxide has the largest cation/anions charge exchange. The result lets suggest that the ionic character introduced by $X$ is less effective than the covalent one introduced by $\mathrm{N}$, whence the expectation of strong Ce- $\mathrm{N}$ as discussed in next section.

\section{Electronic structure and chemical bonding}

With the calculated parameters of the ground state structures (Tables 1 and 2), the detailed electronic band structure and chemical bonding properties are obtained using scalar relativistic calculations with the full potential ASW method [17]. The plots for $\mathrm{CeO}_{2}$ are also presented with parameters from ref. [22]. As above, at self consistent energy convergence the charge transfer is from Ce towards $\mathrm{N}$ and $X$ in CeNX on one hand 
and from $\mathrm{Ce}$ to $\mathrm{O}$ in $\mathrm{CeO}_{2}$ on the other hand, in agreement with the above computed quantities.

Figs. 2 show the electronic band structures along the major directions ( $x$ - axis) of the respective Brillouin zones, i.e. tetragonal, rhombohedral and cubic. The energy along the $y$-axis is with respect to $\mathrm{E}_{\mathrm{V}}$, top of the valence band (VB) in so far that the three compounds are insulating with gap magnitudes of $\sim 1.9 \mathrm{eV}(\mathrm{CeNCl}), \sim 1.8 \mathrm{eV}(\mathrm{CeNF})$ and $\sim 2.2 \mathrm{eV}\left(\mathrm{CeO}_{2}\right)$. The latter gap magnitude agrees with the insulating character identified by SCF methods [22]. The top of VB is dominated by halogen (oxygen) $p$ states and separated by the band gap from the conduction band (CB) containing mainly Ce empty $4 f$ states. However the chemical bonding (quantum mixing) between Ce and $\mathrm{X}(0)$ is ensured by the itinerant parts of $f, d$ and $p$ states respectively at the top of VB, as detailed in the density of states (DOS) plots which also address the shape of the bands. The lower parts of the valence bands comprise the low energy lying $s$-like bands.

Figs. 3 present the site projected DOS (PDOS). They mirror the band structure plots by showing the dominant localized (sharp) Ce states within CB and the broader delocalized states arising from itinerant (red lines) Ce having similar skyline with N-p states (green lines) and less magnitude and similarity with $\mathrm{X}$. These features are encountered in the two panels $3 \mathrm{a}$ and $3 \mathrm{~b}$. On the opposite less mixing of $\mathrm{F}-p$ (blue) can be observed with the rare earth itinerant states. Further they are separated from the Ce$\mathrm{N}$ (red-green) block and found at lower energy in CeNF (Fig. 3b) versus panel 3a (CeNCl) due to the larger electronegativity of $\mathrm{F}$ versus $\mathrm{Cl}$. These DOS are mirrored by the non dispersed bands between -4 and $-6 \mathrm{eV}$ in Fig. $2 \mathrm{~b}$. The mixing between Ce and $\mathrm{N}$ is also observed in the lower part of the VB for the $s$ states. Note that the halogen $s$ states, not shown, are found at energies below $-13 \mathrm{eV}$ with respect to $\mathrm{Ev}$.

Fig. $3 c$ showing the PDOS of $\mathrm{CeO}_{2}$ presents similar features with the other panels regarding the position of Ce empty $4 f$ states within the $\mathrm{CB}$ and the top of the $\mathrm{VB}$ dominated by itinerant $\mathrm{Ce}$ and $\mathrm{O}$ states. This is expected in so far that the three compounds are isoelectronic, meaning that the same numbers of electrons are redistributed over different atomic constituents. The major differences with respect to the nitride-halide DOS arise from the presence of halogen.

The quantum mixing between Ce on one hand and X $(0)$ on the other hand can be further assessed using the COOP criterion based on overlap matrix elements $S_{\mathrm{ij}}$ of 
chemical bonding [23]. In the plots positive, negative and zero intensities correspond to bonding, antibonding and non-bonding interactions. Figs. 4 show the COOP for pair interactions in $\mathrm{CeNCl}$ and $\mathrm{CeNF}$ in both ground state rhombohedral and tetragonal forms for comparisons: Ce-N is bonding throughout the VB in all three first panels although a small anti-bonding contribution (negative magnitudes along COOP y -axis) is observed at $\sim-1.8 \mathrm{eV}$ in $r h$-CeNF (panel $b$ ). However comparing CeNCl and CeNF COOP, it is clear that Ce-N bonding is stronger in the latter, in agreement with the Ce-N smaller separation and the covalent like bonding, contrary to Ce-F larger distance with ionic like bond. These latter are bonding at the energy position of $\mathrm{F}(p)$ in the DOS plot (Fig. 2b) and presenting more intense antibonding COOPs in the $\sim 4 \mathrm{eV}$ energy range below $\mathrm{E}_{\mathrm{V}}$. In hypothetic tetragonal structure of CeNF (Fig. 4c), the bonding Ce-N is largely compensated with antibonding $\mathrm{Ce}-\mathrm{F}$, in agreement with the less stability of the compound in such layered structure.

\section{Conclusions and prospective synthesis route for CeNF}

Based on the fact that the whole ThNX nitride halides exists, with rhombohedral symmetry for $X=\mathrm{F}$ and tetragonal for the other $X=\mathrm{Cl}, \mathrm{Br}$, I, whereas only $\mathrm{CeNCl}$ is identified and (partially) experimentally characterized in tetragonal symmetry, we have investigated both $\mathrm{CeNCl}$ and hitherto undiscovered CeNF within DFT methodology.

The results complement the early crystal structure determinations of $\mathrm{CeNCl}$ and show its stability in the experimental structure, i.e. versus rhombohedral symmetry. This is opposite to CeNF which is clearly identified in the rhombohedral symmetry like ThNF from energy criteria. Similarly to $\mathrm{CeO}_{2}$, both $\mathrm{CeNF}$ and $\mathrm{CeNCl}$ are insulating with smaller band gaps thanks to the $\mathrm{Ce}-\mathrm{N}$ covalent bond. This prevailing bond was also identified from analyses of overlap populations.

The computational results let propose synthetic routes presently under evaluation along two different protocols:

a- the 'ammonolysis' of the fluoride $\mathrm{Ce}^{\mathrm{III}} \mathrm{F}_{3}$ precursor such as the protocol used by Schurz et al. for obtaining MNX family [8] or ;

b- the method using fluorinated fluxes, preferably with nascent fluorine (strongly oxidizing) on CrIIIN following a protocol proposed for preparing ZrNF [5]. 
The latter protocol seems relevant from the electronic structure characteristics of CeNF, whereby within CeN [24], covalent Ce-N bonding is expected to be modified but still keep prevailing as shown from the COOP analysis upon introducing fluorine. Lastly the reaction CeN $+1 / 2 \mathrm{~F}_{2} \rightarrow$ CeNF is calculated favorable for synthesis with negative enthalpy of $\sim-1.6 \mathrm{eV} /$ at.

Acknowledgments: The author is grateful to Dr Alain Demourgues (ICMCB-CNRS) for discussion on the topic of CeNX at an early stage of the preparation of the manuscript. Support from Conseil Régional d'Aquitaine is acknowledged. Parts of the computations were carried out on the computers of the MCIA-Université de Bordeaux. 


\section{References}

[1] J.L. Soubeyroux, S.F. Matar, J.M. Reau, P. Hagenmuller, Solid State Ionics, 14, 337, (1984).

[2] H Zhong-Zhen Luo, Chen-Sheng Lin, Wei-Long Zhang, Hao Zhang, Zhang-Zhen He and Wen-Dan Cheng, Cryst. Eng. Comm, 16, 2788 (2014).

[3] M.J. Xia, R.K. Li, Solid State Comm. 201, 102 (2015).

[4] C. Wüstefeld, Th. Vogt, U. Löchner, J. Strähle, H. Fuess, Angew. Chemie Int. Ed. in English, 27, 929 (1988).

[5] L. Zhu, X. Chen, Sh. Yamanaka, Solid State Communications 130, 227 (2004).

[7] R. Juza, R. Sievers, Z. Anorg. Allg. Chem., 363, 258 (1968).

[8] Ch. M. Schurz, L. Shlyk, Th. Schleid, R. Niewa, Z. Kristallogr., 226, 395 (2011).

[9] W. Kohn, L.J. Sham, Phys. Rev. A, 1401133 (1964).

[10] P. Hohenberg, W. Kohn, Phys. Rev. B, 136864 (1965).

[11] S.F. Matar, G. Demazeau, Chem. Phys. Lett. 498, 77 (2010).

[12] S. F. Matar, J. Solid State Chem., 185, 25 (2012).

[13] S. F. Matar, M. Pouchard, Solid State Sci. 18, 123 (2013)

[14] S. F. Matar, J. Magn. Magn. Mater. 368, 105 (2014)

[15] G. Kresse, J. Furthmüller, Phys. Rev. B, 54, 11169 (1996).

[16] G. Kresse, J. Joubert, Phys. Rev. B, 59, 1758 (1999).

[17] V. Eyert, V. The Augmented Spherical Wave Method - A Comprehensive Treatment, In: Lecture Notes in Physics, Springer, Berlin, Heidelberg, 2007, chapter 719.

[18] J. Perdew, K. Burke, M. Ernzerhof, Phys. Rev. Lett., 77, 3865 (1996).

[19] S. F. Matar, Prog. Solid State Chem., 38, 1 (2010).

[20] E.A. Mason, E.W. Mc Daniel, Trnasport Properties of Ions and Gases. (C) 1988 by John Wiley and Sond, Inc. ISMB: 0-471-88385-9.

[21] R. Bader, Chem. Rev. 91, 893 (1991).

[22] D.D. Koelling, A.M. Boring, J.H. Wood, Solid State Commun. 47, 227 (1983).

[23] R. Hoffmann, Angew. Chem. Int. Ed. Engl. 26, 846 (1987).

[24] A. Mavromaras, S.F. Matar, B. Siberchicot, G. Demazeau, J. Magn . Magn . Mater., 134, 34 (1994). 
Table 1.

CeNCl and CeNF in PbFCl-type structure $(P 4 / \mathrm{nmm})$ : Calculated and (experimental) lattice and atomic parameters in tetragonal symmetry. Unchanged $\mathrm{N}$ position at origin $(0,0,0)$. FU: formula unit. Starting parameters for CeNF are those of CeNCl.

\begin{tabular}{|c|c|c|}
\hline$P 4 / n m m(Z=2 \mathrm{FU})$ & $\mathrm{CeNCl}$ & CeNF \\
\hline $\mathrm{a}(\AA)$ & $4.01(4.079)$ & 3.797 \\
\hline $\mathrm{c}(\AA)$ & $6.93(6.837)$ & 5.881 \\
\hline $\mathrm{V}\left(\AA^{3}\right)$ & $111.4(113.76)$ & 84.79 \\
\hline \multicolumn{3}{|l|}{ Atoms at $(2 c) 0,1 / 2, z$} \\
\hline$Z_{\mathrm{Ce}}$ & $0.166(-)$ & 0.203 \\
\hline$Z_{\mathrm{Cl}}$ & $0.628(-)$ & 0.605 \\
\hline $\begin{array}{l}\mathrm{d}(\mathrm{Ce}-\mathrm{N})(\AA) \\
\mathrm{d}(\mathrm{Ce}-X)(\AA)\end{array}$ & $\begin{array}{l}2.30(2.33) \\
3.18(3.20) \\
\end{array}$ & $\begin{array}{l}2.24 \\
2.37 \\
\end{array}$ \\
\hline Energy $(\mathrm{eV})$ & -43.34 & -47.13 \\
\hline
\end{tabular}

Table 2

$\mathrm{CeNCl}$ and CeNF in rhombohedral $R \overline{3} m$ ThNF structure: Calculated lattice and atomic parameters with ThNF starting parameters.

N.B. $a_{\text {hex. }}=a_{\mathrm{R}} \sqrt{ }\left(2(1-\cos \alpha) ; c_{\text {hex. }}=a_{\mathrm{R}} \sqrt{ } 3(1+2 \cos \alpha)\right.$, i.e. $a=3.878 \AA, c=19.928 \AA$

\begin{tabular}{|c|c|c|}
\hline$R \overline{3} m(Z=2 \mathrm{FU})$ & CeNCl & CeNF \\
\hline $\begin{array}{l}a(\AA) \\
\alpha_{\text {rh. }}\end{array}$ & $\mid \begin{array}{l}8.51 \\
27^{\circ} 01^{\prime}\end{array}$ & $\begin{array}{l}7.01 \\
32^{\circ} 12^{\prime}\end{array}$ \\
\hline $\mathrm{V}\left(\AA^{3}\right)$ & 102.50 & 84.82 \\
\hline \multicolumn{3}{|c|}{ Atoms at $(2 c) x, x, x$} \\
\hline$X_{\mathrm{Ce}}$ & 0.223 & 0.261 \\
\hline$X_{\mathrm{N}}$ & 0.378 & 0.377 \\
\hline$X_{\mathrm{F}}$ & 0.132 & 0.131 \\
\hline $\begin{array}{l}\mathrm{d}(\mathrm{Ce}-\mathrm{N})(\AA) \\
\mathrm{d}(\mathrm{Ce}-X)(\AA)\end{array}$ & $\begin{array}{l}2.20 \\
2.82 \\
\end{array}$ & $\begin{array}{l}2.37 \\
2.61 \\
\end{array}$ \\
\hline Energy $(\mathrm{eV})$ & -43.11 & -47.78 \\
\hline
\end{tabular}




\section{FIGURES}

Fig. 1. Tetragonal CeNCl and rhombohedral CeNF ground state structures.

Fig. 2. Electronic band structure along major respective Brillouin zones of $\mathrm{CeNCl}$, CeNF and $\mathrm{CeO}_{2}$.

Fig.3. Site projected DOS for CeNCl, $\mathrm{CeNF}$ and $\mathrm{CeO}_{2}$.

Fig. 4. Chemical bonding for pair interactions in $\mathrm{CeNCl}$, $\mathrm{CeNF}$ and $\mathrm{CeO}_{2}$. 


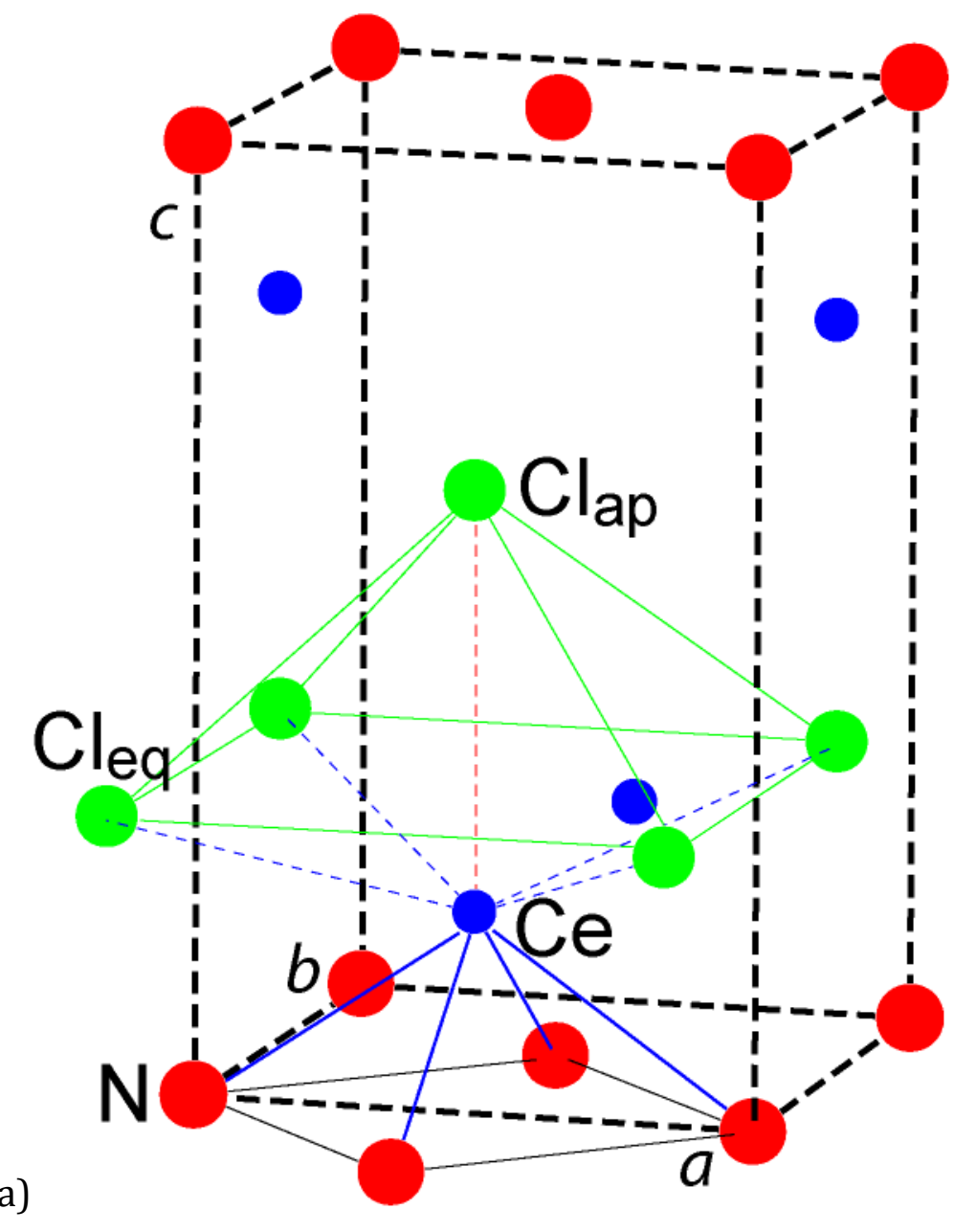




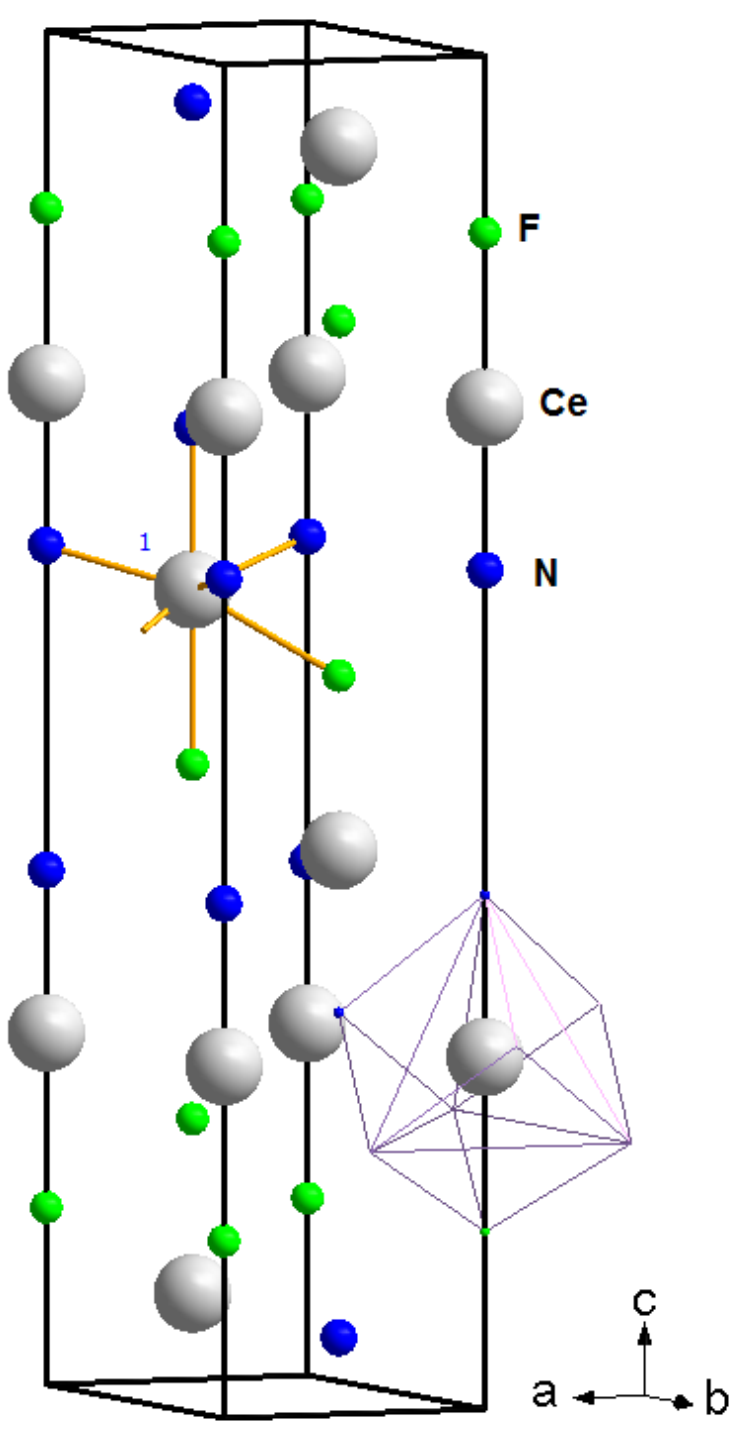

b)

Fig. 1. 

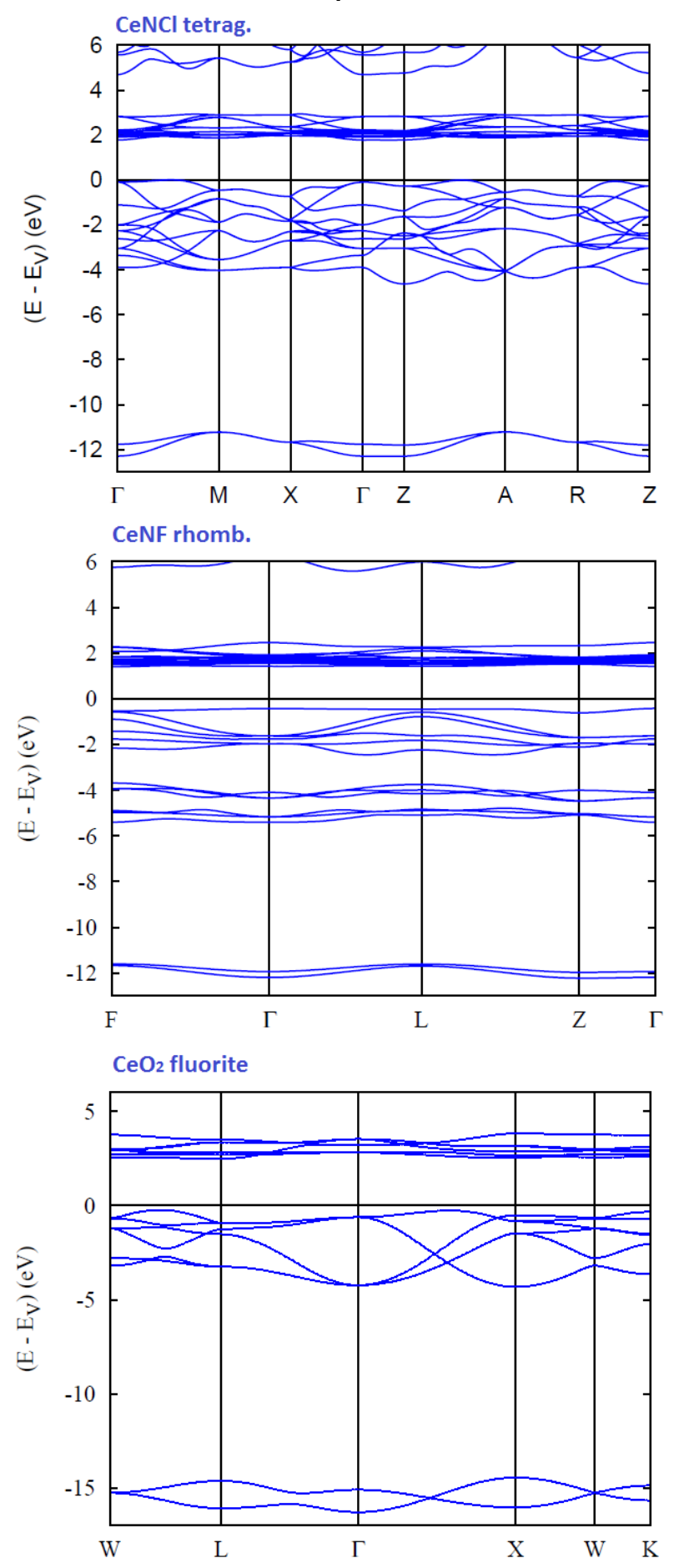

Fig. 2 

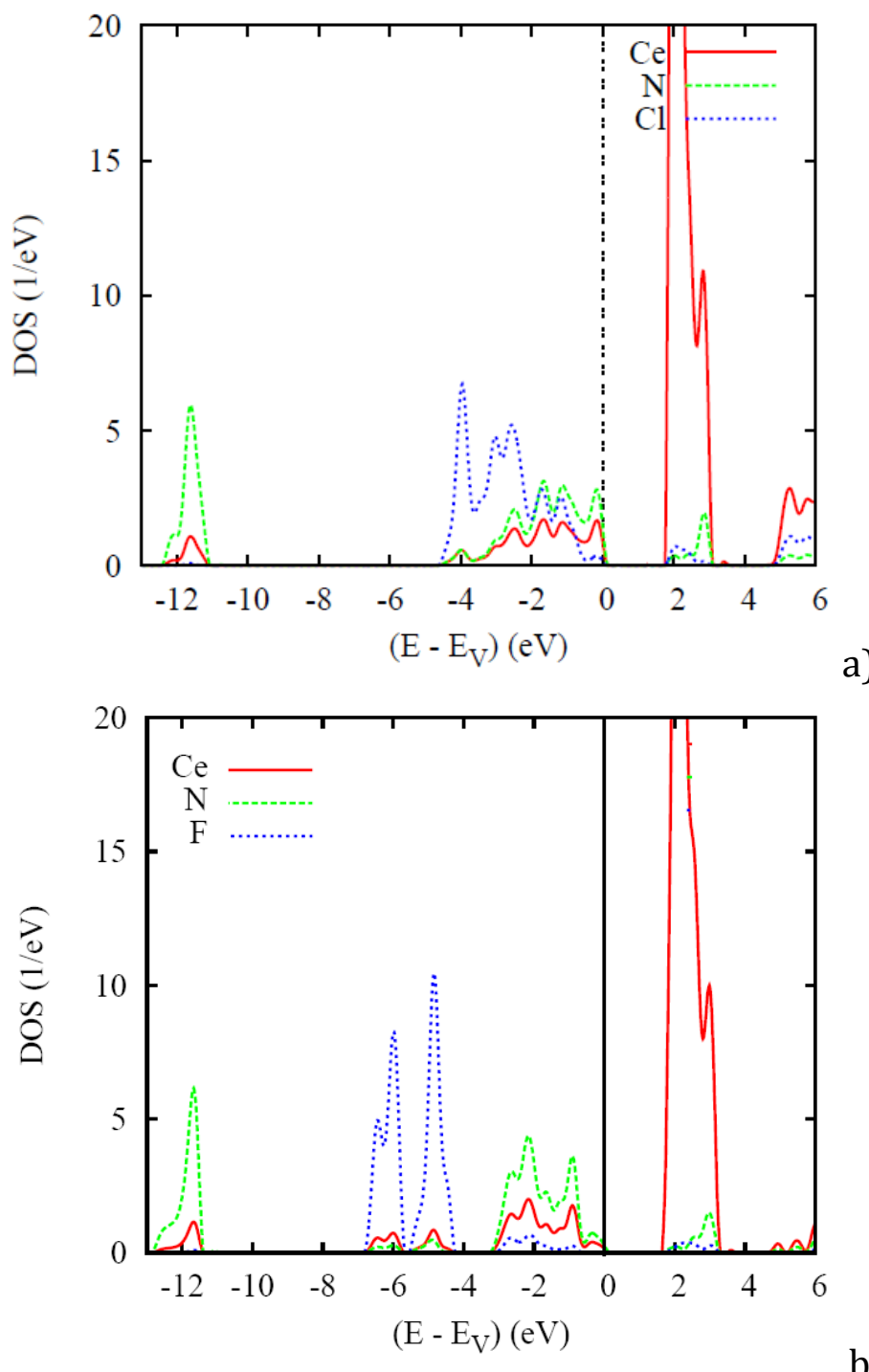

b)

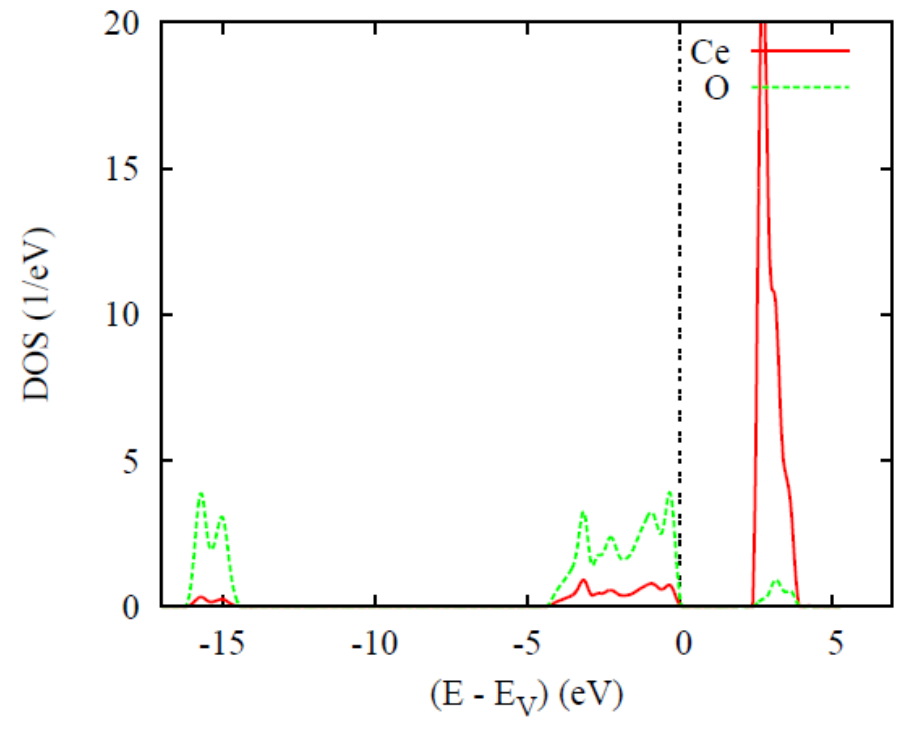

c)

Fig.3 


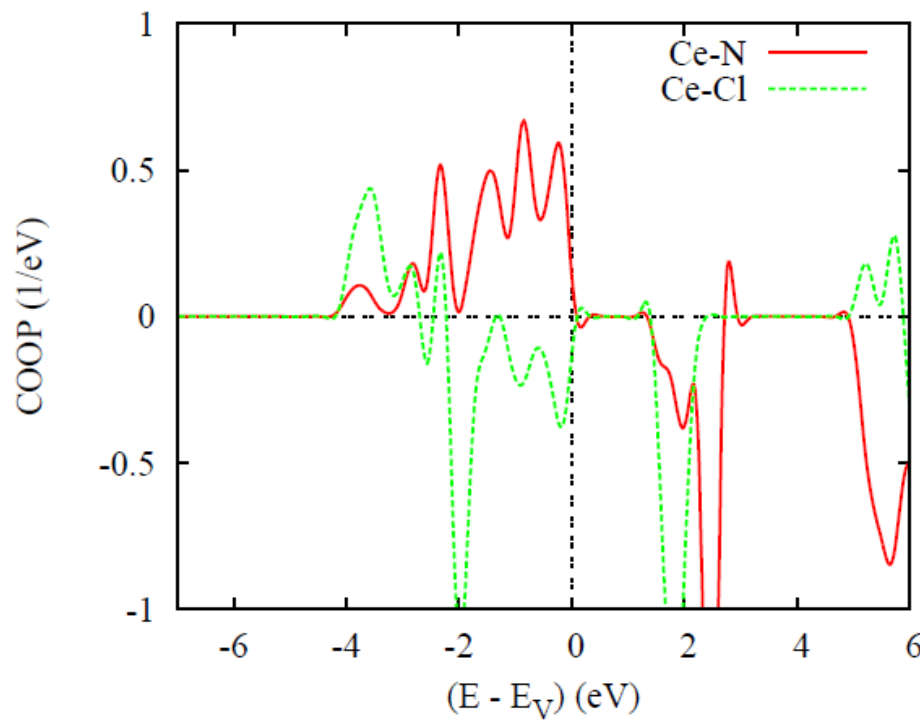

a)

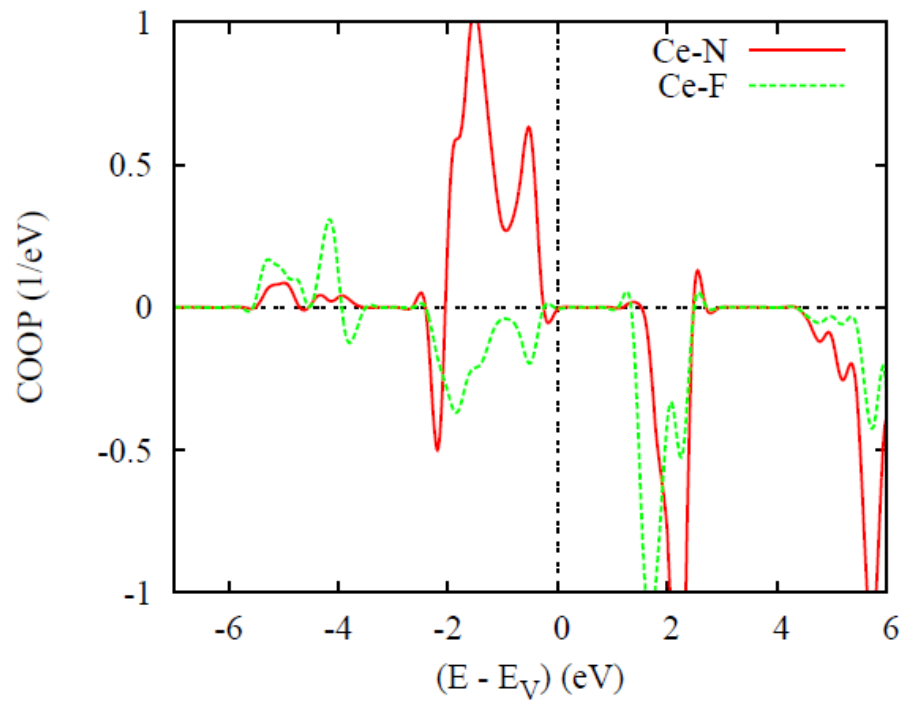

b)

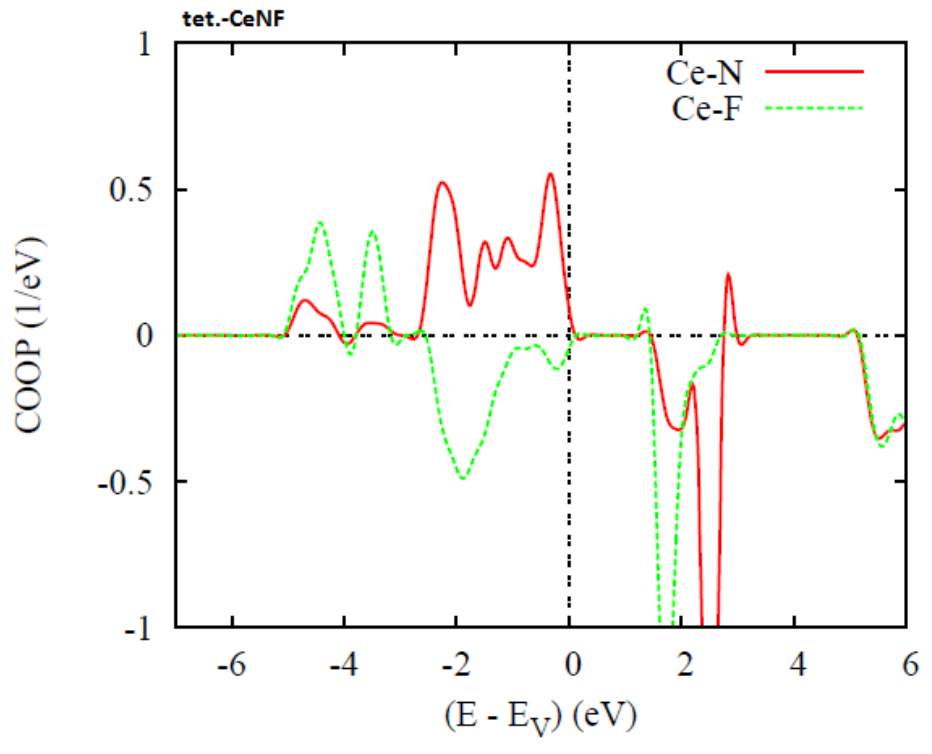

Fig. 4. 